\title{
TA-PROJEKTE
}

\section{Big Data und die gesellschaftlichen Folgen}

\begin{abstract}
von Carsten Orwat, Reinhard Heil, Anika Hügle, René König und Christina Merz, Institut für Technikfolgenabschätzung und Systemanalyse (ITAS), Karlsruhe
\end{abstract}

Die Erzeugung, Verknüpfung und Auswertung großer Datenmengen, oft mit dem Begriff „Big Data“ verbunden, beeinflusst in unserer modernen, digitalisierten Gesellschaft beinahe alle Lebensbereiche. Vernetzte Computertechnologie, Smartphones, soziale Netzwerke, vielfältige Online-Angebote und die Durchdringung des Alltags mit dem ,Internet der Dinge“ führen zu einer rasanten Vervielfachung von Datenquellen und Datenmengen. Neben den Potenzialen von Big Data, z. B. in den Bereichen Wirtschaft oder Medizin, werden zunehmend auch die gesellschaftlichen Herausforderungen deutlich. Es ergeben sich insbesondere Fragen zu kommerzieller und staatlicher Überwachung, informationeller Selbstbestimmung, Schutz der Privatsphäre, Transparenz bei den Verfahren der Datenverarbeitung oder zu Fehlern bei Datenverwendungen sowie automatisierten Entscheidungen. Das Projekt ABIDA (Assessing Big Data - Begleitforschung Big Data) verfolgt das Ziel, Wissen aus gesellschaftlicher Perspektive über Entwicklungen, Herausforderungen und Handlungsoptionen von Big Data zusammenzubringen, zu erzeugen, zu vertiefen und zu verbreiten. ${ }^{1}$

\section{Technologien und Anwendungen}

\subsection{Unklarer und mehrschichtiger Begriff}

Eine allgemein anerkannte und geteilte Definition von Big Data existiert bisher nicht. Im engeren Sinne werden unter Big Data-Technologien ein Reihe von Anwendungen der Statistik, des maschinellen Lernens bzw. der Mustererkennung wie Korrelationen, Anomalien und Trends gefasst sowie vor allem Programme und Verfahren zur Handhabung und Auswertung von großen Datenmengen (z. B. Apache Hadoop, Google MapReduce oder Apache Flink), insbesondere dort, wo bisherige Datenverarbeitungstechnologien nicht mehr ausreichen (z. B. Chen 2014).

Über die enge, pragmatische Begriffsfassung hinaus ist Big Data mittlerweile mit Bestrebungen verknüpft, mittels immer mehr, weitgehend automatisierten Datenerzeugungen und -auswertungen zu umfassenderen Analysen, Kategorisierungen, Vorhersagen und letztlich auch zu Kontrollen von Zuständen, Entwicklungen von Menschen, ihren Handlungen und Interaktionen, Dingen und Umwelten zu gelangen. Dabei wird oft auf die Verknüpfung bislang unverbundener Datensätze und die Realisierung von Analysen in „Echtzeit“, d. h. mit zeitlich unmittelbarer Zurverfügungstellung und Reaktionsmöglichkeiten, abgezielt. Eng verknüpft sind damit auch neue Praktiken und Geschäftsmodelle der Weiterverwertung, des Handels sowie der Verknüpfung, Aggregation und Analyse von Daten, nicht nur in Wissenschaft oder Wirtschaft, sondern auch im staatlichen und öffentlichen Bereich.

Als das charakteristisch Neue an Big Data werden die mit den Datenmengen und deren Detaildichte entstehenden Formen der Erkenntnisgewinnung gesehen. Bei den Beobachtungs- und Untersuchungsobjekten können in den großen Datenmengen automatisiert nach Korrelationen gesucht werden, die zu neuen Hypothesenbildungen anregen. Die Begrenztheit von Zufallsstichproben wird dadurch überwunden, dass vielfältige Daten zu den jeweiligen Objekten Analysen aus verschiedenen Perspektiven und hinsichtlich unterschiedlichster Details ermöglichen (MeyerSchönberger 2015).

\subsection{Gegenwärtige Vervielfachung der Datenquellen und Datenmengen}

Gegenwärtig findet eine Vielzahl von gleichzeitigen Entwicklungen statt, die dazu führen, dass Daten nicht nur in der Quantität sehr schnell wachsen, sondern auch hinsichtlich der erfassten Lebensbereiche sowie der Detailgenauigkeit zunehmen. Grundlegend ist die seit mehreren Jahrzehnten stattfindende massenhafte Digitalisierung von Informationen und die Elektronisierung von Interaktionen, die heute auch Bezahlsysteme (z. B. Bitcoin oder Apple Pay, elektronische Tickets im ÖPNV) und sensible Informationen wie 
Patientenakten oder genetischen Informationen umfasst. Zudem scheint die fortlaufende Verlagerung der sozialen Kommunikation und Aktivitäten in Onlinebereiche, von der Suche nach Informationen, über die Kommunikation in sozialen Gruppen, die Online-Inanspruchnahme von Speicher- und Softwarediensten (,cloud computing"), das Einkaufen von Konsumwaren, bis hin zur Transport-, Übernachtungs- oder Partnersuche, weiterhin ungebremst. Die dabei erfolgende Erfassung nicht nur der Verbindungsdaten, sondern auch der Kommunikationsinhalte, Interaktionen und Transaktionen kann als eine der Hauptquellen von Big Data angesehen werden.

Neu hinzu kommt die massenhafte Einbettung digitaler und vernetzter Informations- und Kommunikationstechnologien, v. a. bedingt durch kostengünstige Sensoren und Rechenkapazitäten, in Dingen aus vielfältigsten Lebensbereichen von Häusern, über Verkehrsmittel, Infrastruktureinrichtungen, Produktions- und Arbeitsstätten, Konsumwaren bis hin zu Freizeitgegenständen. Derartige Entwicklungen werden gerne unter Begriffe wie „Internet der Dinge und Dienste“, „Industrie 4.0", „smart cities“, „smart homes“, „smart grid“" und „wearables“ subsumiert. Da dabei Daten in den unmittelbaren, öffentlichen wie privaten Lebensumgebungen von Menschen erzeugt werden, sind sie meist personenbezogen oder leicht personenbeziehbar. Dieser Trend resultiert auch aus der fortlaufenden Ergänzung und Verlagerung von kabelgebundener zur mobilen Kommunikation, bei der neben den Daten über digitale Kommunikationsverbindungen und -inhalten auch gleichzeitig Daten über Standorte, Bewegungsprofile und Verhaltensmuster von Individuen erzeugt werden. Die dadurch ermöglichte Lokalisierung geht in der Regel einher mit der Personalisierung der Dienste und Produktangebote und somit der Erzeugung eben jener personenbezogenen oder personenbeziehbaren Daten. Verstärkt wird diese Entwicklung zusätzlich noch durch die zunehmende Anwendung von digitalen Mitteln der Personenidentifizierung und des Identitätsmanagements, wie Online-Identitätsmanagement oder elektronische Zugangssysteme, oder die mit Zwang eingeführten Systeme der elektronischen Gesundheitskarte, Mautsysteme oder biometrischen Systemen an Grenzkontrollen.

\subsection{Ein kurzer Blick auf gesellschaftliche Veränderungen}

All diese Entwicklungen führen dazu, dass Daten in bisher nicht gekanntem Ausmaß und Granularität anfallen. Die vielfältigen gesellschaftlichen Implikationen lassen sich an dieser Stelle nur sehr kurz betrachten, ihnen ist jedoch das Hauptaugenmerk des ABIDA-Projekts gewidmet.

Deutlich wird bereits heute, dass Big DataAnwendungen in ihrer gesellschaftlichen Relevanz vor allem dahingehend $\mathrm{zu}$ unterscheiden sind, ob durch sie Daten mit konkretem oder ableitbarem Personenbezug erzeugt und analysiert werden oder nicht. In vielen Anwendungsbeispielen der Wirtschaft fallen Daten zu Produktionsanlagen, Prozessen, Produkten, Zulieferern etc. ohne Personenbezug an (z. B. BITKOM 2015). Big Data-Anwendungen für Vorhersagen von Produktionsstörungen oder Lieferausfällen und Reaktionen in Echtzeit sind dabei jedoch anders zu beurteilen als Beispiele aus anderen Wirtschaftsbereichen. Denn im Marketingbereich (Big Data für Werbungoptimierung, Preisdifferenzierung oder Kundenmanagement) oder im Finanz- und Versicherungssektor (flexible Versicherungstarife oder Kreditscoring) fallen sensible Daten an, die zu teil- oder vollautomatisierten Kategorisierung und Bewertungen von Individuen genutzt werden (z. B. Citron 2014; Rothmann 2014) und bis hin zum ,scoring“ von Kunden, Mietern, Beschäftigten und potenziellen Arbeitnehmern führen können.

So deuten sich in vielen Fällen des personenbezogenen Big Data Spannungen zu den bewährten Grundprinzipien des Datenschutzes, insbesondere der Datensparsamkeit und Zweckbindung, an. Beim Scoring von Arbeitnehmern fließen beispielsweise nicht nur zweckgebundene Daten, wie Arbeitnehmerstammdaten oder Fehlzeiten, sondern auch Auswertungen von Daten aus sozialen Netzwerken in die Bewertungen mit ein (LinkedIn 2015). Auch die Datenschutzprinzipien Informiertheit über und Einverständnis des Nutzers zu Datenerzeugungen und -verwendungen scheint angesichts der kaum von außen nachvollziehbaren unternehmensinternen und unternehmensübergreifenden Datenweiterverwendung und Weitergaben an Grenzen zu stoßen, nicht zuletzt auch weil individuelle Kapazitäten, 
die Vielzahl an juristisch anspruchsvoll formulierten, allgemeinen Geschäftsbedingungen vor Zustimmung notwendigerweise zu verarbeiten, schlichtweg begrenzt sind (Acquisti et al. 2015). Weitere Datenschutzprobleme, wie die der RePersonalisierbarkeit durch Kombination eigentlich anonymisierter Daten (Ohm 2010), sind noch gänzlich ungelöst. Ferner müssen Abschätzungen der Implikationen von Big Data hinsichtlich Datenschutz und informationeller Selbstbestimmung auch vor dem Hintergrund aktueller Reformen des Datenschutzrechts, insbesondere zur EU-Datenschutz-Grundverordnung sowie den Handelsabkommen TTIP ${ }^{2}$ und TiSA2 ${ }^{3}$, erfolgen, ebenso im Hinblick auf Verunsicherungen in der Bevölkerung, die durch Skandale staatlicher Überwachung ausgelöst worden sind.

Neben den Datenschutzfragen zeigen sich weitere gesellschaftliche Problembereiche wie der des Verbraucherschutzes. Da Big Data auch zur Kategorisierung und Differenzierung von Personen und Personengruppen eingesetzt wird, etwa zur Preisdifferenzierung auf Warenmärkten, kann es zu ungerechtfertigten Preisdifferenzierungen bzw. Diskriminierungen kommen (US CEA 2015). Tendenzen der Konzentration in der Datenerzeugung und -haltung werfen auch wettbewerbspolitische Fragen und Fragen zum politischen Umgang mit Machtverschiebungen auf.

\subsection{Zusammenführen disziplinären Wissens}

Die Anzahl der wissenschaftlichen Publikationen und Veranstaltungen zu Big Data ist stark angestiegen; oft werden Fragestellungen und Forschungsrichtungen aus disziplinärer Sicht erst entwickelt oder es wird sich auf allgemeine Reflexionen oder Fallstudien beschränkt. Um dieses verstreute Wissen der Geistes- und Gesellschaftswissenschaften zusammenzuführen und weiterzuentwickeln, werden Arbeitskreise in den Disziplinen Recht (geleitet von Nikolaus Forgó, Hannover), Ethik (geleitet von Klaus Wiegerling, ITAS), Ökonomie (geleitet von Arnold Picot, München), Soziologie (geleitet von Johannes Weyer, Dortmund) und Politik (geleitet von Jeanette Hofmann, Berlin) betrieben und eine Fachtagung (15.-16.2.16 in Münster) abgehalten.

\subsection{Beobachtung und Erfassung gegenwärtiger Entwicklungen}

Abgesehen von einigen, in den Medien stark präsenten Beispielen, wie die Verwendung von mit Fitness-Armbändern erhobenen Vitaldaten in neuen, ,interaktiven“" Tarifmodellen einiger Krankenkassen, zeigen sich die vielfältigen Big Data-Anwendungen nur punktuell und wenn, dann nur eher Fachkreisen. Das Bild, wie, wo und zu welchen Zwecken große Datenmengen erhoben, verarbeitet, gehandelt oder verknüpft wer-

\section{Das Projekt ABIDA}

Um das Projektziel zu erreichen, aus gesellschaftlicher Perspektive Wissen über Entwicklungen, Herausforderungen und Handlungsoptionen von Big Data zusammenzutragen, zu erzeugen, zu vertiefen und $\mathrm{zu}$ verbreiten, werden im Projekt eine Reihe von zusammenwirkenden, in vier Schwerpunkte gliederbare Forschungs- und Dialoginstrumente der Technikfolgenabschätzung angewandt (Abb. 1).
Abb. 1: ABIDA-Projektstruktur

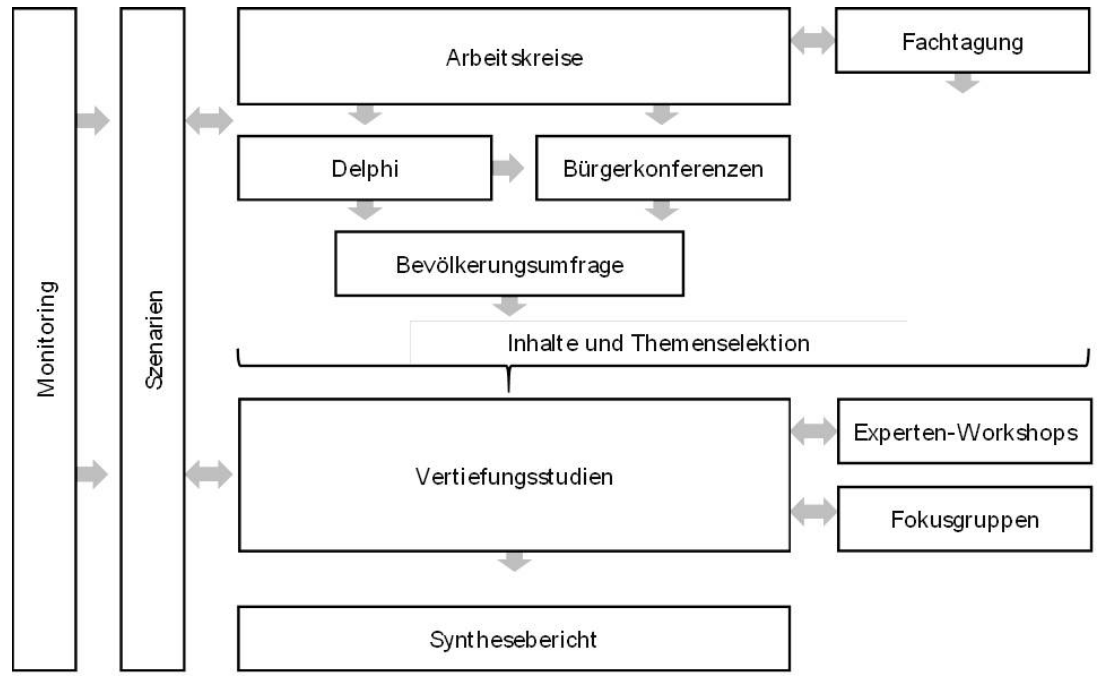

Quelle: Eigene Darstellung 
den, ist bisher noch weitestgehend unklar. Eine der Aufgaben des Projekts ABIDA ist es daher, Erkenntnisse über gegenwärtige und geplante Anwendungen von Big Data zusammenzutragen und zu vertiefen. Dazu werden die Entwicklungen bei Big Data-Technologien, -Anwendungsmodellen, -Problembereichen und -Rahmenbedingungen mithilfe eines kontinuierlichen Monitorings erfasst, beobachtet und Bewertungen zugeführt.

Des Weiteren ergeben sich viele Forschungsfragen, die sich auf die durch Big Data hervorgerufenen Veränderungen in einzelnen Wirtschaftssektoren beziehen, wie sich dies etwa in der Medizin, im Finanzsektor oder im Verkehr abzeichnet. Diese Veränderungen sollen mit Vertiefungsstudien zu ausgewählten, für Big Data besonders relevante Branchen und Themen abgeschätzt werden. Dies schließt die Gewinnung von Fachexpertise durch externe Gutachten mit ein. Ergänzende Fachkenntnisse und Einschätzungen sollen mit Hilfe von Expertenworkshops und Fokusgruppen zu den ausgewählten Branchen und Themen erhoben werden, bei denen Vertreter von Interessengruppen und Betroffenen involviert werden sollen.

\subsection{Ermittlung der Sichtweisen der Bürgerinnen und Bürger}

Was wissen Bürgerinnen und Bürger über Big Data und die hinter dem Begriff stehenden Anwendungen oder Geschäftspraktiken? Was wissen oder vermuten sie über ihren Nutzen und ihre Risiken? Wie nutzen sie bereits Vorteile konkret oder gehen mit möglichen Gefährdungen um, ergreifen etwa „selbstschützende“ Maßnahmen? Um die Sichtweisen von Bürgerinnen und Bürgern auf Potenziale und Herausforderungen von Big Data zu ermitteln, werden nicht nur drei Bürgerkonferenzen an verschiedenen Standorten in Deutschland durchgeführt, sondern auch eine ergänzende, repräsentative Bevölkerungsumfrage.

\subsection{Abschätzung von künftigen Entwick- lungen und Handlungsoptionen}

Im Sinne der Technikfolgenabschätzung sollen möglichst frühzeitig die mit Big Data zu erwartenden gesellschaftlichen Veränderungen erkannt und abgeschätzt werden. Dadurch soll beispielsweise bei potenziellen Fehlentwicklungen, wie bei sich abzeichnenden ethischen oder rechtlichen Konflikten, noch vor ihrer Konkretisierung oder gar Verfestigung mit geeigneten Maßnahmen reagiert werden können. Zu diesem Zweck sind in ABIDA entsprechende Projektelemente vorgesehen, insbesondere die Erstellung von Szenarien sowie die Durchführung einer DelphiStudie. Letztere beinhaltet sowohl eine OnlineBefragung von Experten und Interessenvertretern als auch einen Delphi-Workshop. Neben der Diskussion und Abschätzung künftiger Entwicklungen geht es bei diesen Instrumenten auch darum, Alternativen zu gegenwärtigen und absehbaren Entwicklungen aufzuzeigen und zu diskutieren. Als Resultat sollen insbesondere Handlungsoptionen erarbeitet werden. Dazu zählen vor allem auch Optionen der politischen Steuerung und der Governance, die neben der im Mittelpunkt stehenden Gestaltung des künftigen Datenschutzes, auch Optionen der Verbraucher- und Wettbewerbspolitik sowie der Technologie- und Innovationspolitik umfassen können. So können auch Fragestellungen in den Fokus rücken, wie die Souveränität und Entscheidungsräume der Betroffenen bzw. Endnutzer sowie deren Kontrolle über Datenverwendung und Identitätsbildung zurückgewonnen und gesichert werden können oder wie man vertrauensvolle Infrastrukturen der Big Data-Nutzung schaffen und betreiben kann.

\section{Anmerkungen}

1) Das Projekt ABIDA wird vom Bundesministerium für Bildung und Forschung (BMBF) von März 2015 bis Februar 2019 gefördert (Förderkennzeichen 01IS15016A-F). Zusammen mit dem Institut für Informations-, Telekommunikations- und Medienrecht (ITM) der Westfälischen Wilhelms-Universität Münster koordiniert und betreibt das ITAS das Verbundprojekt. Weitere Partner zur Durchführung der Arbeitskreise sind das Institut für Techniksoziologie der Technischen Universität Dortmund, das Institut für Rechtsinformatik der Gottfried Wilhelm Leibniz Universität Hannover, die Forschungsstelle für Information, Organisation und Management der Ludwig-Maximilians-Universität München sowie das Wissenschaftszentrum Berlin für Sozialforschung. Weitere Informationen zum Projekt können im Web unter http://www.abida.de abgerufen werden. 
2) TTIP: Transatlantic Trade and Investment Partnership (Transatlantische Handels- und Investitionspartnerschaft)

3) TiSA: Trade in Services Agreement (Abkommen über den Handel mit Dienstleistungen)

\section{Literatur}

Acquisti, A.; Brandimarte, L.; Loewenstein, G., 2015: Privacy and Human Behavior in the Age of Information. In: Science 347/6221 (2015), S. 509-514

BITKOM - Bundesverband Informationswirtschaft, Telekommunikation und neue Medien e.V., 2015: Big Data und Geschäftsmodell-Innovationen in der Praxis: 40+ Beispiele. Leitfaden. Berlin

Chen, M.; Mao, S.; Liu, Y., 2014: Big Data: A Survey. In: Mobile Networks and Applications 19/2 (2014), S. 171-209

Citron, D.K.; Pasquale, F., 2014: The Scored Society: Due Process for Automated Predictions. In: Washington Law Review 89 (2014), S. 101-133

LinkedIn; BITKOM, 2015: Big Data im Personalmanagement. Ergebnisse Unternehmensbefragung. Bitkom Research GmbH im Auftrag von LinkedIn Deutschland, Österreich, Schweiz. München, Berlin

Meyer-Schönberger, V., 2015: Was ist Big Data? Zur Beschleunigung des menschlichen Erkenntnisprozesses. In: Aus Politik und Zeitgeschichte 65/1112 (2015), S. 14-19

Ohm, P., 2010: Broken Promises of Privacy: Responding to the Surprising Failure of Anonymization. In: UCLA Law Review 57/6 (2010), S. 1701-1777

Rothmann, R.; Sterbik-Lamina, J.; Peissl, W., 2014: Credit Scoring in Österreich. Institut für Technikfolgen-Abschätzung der Österreichischen Akademie der Wissenschaften Projektbericht Nr. A66. Wien

US CEA - Council of Economic Advisers, Executive Office of the President of the United States, 2015: Big Data and Differential Pricing. Washington D.C.

\section{Kontakt}

Dr. Carsten Orwat

E-Mail: orwat@kit.edu

Reinhard Heil, M.A.

E-Mail: reinhard.heil@kit.edu

Institut für Technikfolgenabschätzung und

Systemanalyse (ITAS)

Karlsruher Institut für Technologie (KIT)

Karlstr. 11, 76133 Karlsruhe

\section{Wasserstoff im Tank}

\author{
Vorstellung des interdisziplinären \\ Begleitforschungsprojekts zum \\ 50-Wasserstofftankstellen-Programm
}

von Jörg Burkhardt und Andreas Patyk, Institut für Technikfolgenabschätzung und Systemanalyse (ITAS), Karlsruhe

Zur Senkung von Klimagasemissionen und zur Ressourcenschonung wird der Einsatz von Wasserstoff im Mobilitätssektor heute als eine der aussichtsreichsten Alternativen zu fossilen Treibstoffen gesehen. Um die Markteinführung zu stützen, sollen bis Sommer 2016 fünfzig Wasserstofftankstellen in Deutschland errichtet werden. Begleitend zum Tankstellenausbau soll die Technik in dem hier vorgestellten Projekt „Begleitforschung 50-Tankstellen-Programm im Rahmen des Nationalen Innovationsprogramms Wasserstoff- und Brennstoffzellentechnologie" interdisziplinär erforscht werden. So wird neben der Standfestigkeit der Technik z. B. auch ihre Akzeptanz bei Nutzern sowie das energiewirtschaftliche Umfeld analysiert und bewertet. Aus der Sachstandserhebung sollen eventuelle Probleme und Schwachstellen identifiziert und Verbesserungsansätze abgeleitet werden. Das Projekt läuft bis Dezember 2016.

\section{Projektkontext}

Wasserstoff dient im Fahrzeug als Energieträger der meist gasförmig und unter hohen Drücken in dafür vorgesehenen Tankstellen aufgefüllt werden kann. Die erzielbaren Tankzeiten und Fahrzeugreichweiten sind bereits heute mit denen konventioneller Kraftstoffe (Benzin, Diesel) vergleichbar, was ein großer Vorteil der Wasserstoffmobilität ist. Zwar wird Wasserstoff heute überwiegend aus fossilen Energieträgern (Erdgas, Erdöl) hergestellt und in der Chemieindustrie eingesetzt (Smolinka et al. 2011). Da Wasserstoff $\left(\mathrm{H}_{2}\right)$ jedoch auch über Wasserelektrolyse ${ }^{1}$ hergestellt werden kann, gewinnt seine Erzeugung über Strom aus erneuerbaren Energien zunehmend an Bedeutung. Besonders im Rahmen der Energiewende wird die Produktion von Wasserstoff aus überschüssigen Strommengen (z. B. aus Windenergieanlagen) diskutiert. Dessen Nutzung in Wasserstofffahr- 